\title{
Effects of Glyceroglycolipids Prepared from Various Natural Materials on Polymorphic Transformation of Cocoa Butter
}

\author{
Takashi NAKAE, Takashi KOMETANI, Takahisa NISHIMURA, Hiroshi TAKII and Shigetaka OKADA \\ Biochemical Research Laboratory, Ezaki Glico Co. Ltd., Utajima 4-6-5, Nishiyodogawa, Osaka 555-8502, Japan
}

Received June 12, 2000; Accepted August 17, 2000

\begin{abstract}
Monogalactosyldiacylglycerol (MGDG), digalactosyldiacylglycerol (DGDG) and trigalactosyldiacylglycerol (TGDG) were prepared from various natural materials. In all glyceroglycolipids, linoleic acid, linolenic acid, palmitic acid, oleic acid and stearic acid were the major components. The kinetics of polymorphic transformation on cocoa butter and the effect caused by the presence of the glyceroglycolipids prepared from carrot, corn, wheat flour and Chlorella pyrenoidosa were investigated. Each glyceroglycolipid can be incorporated at the level of $5 \%$ within the cocoa butter without changing the crystal lattice. The polymorphic transformation on cocoa butter was induced by continuous temperature cycling between 32 and $20^{\circ} \mathrm{C}$ at $12 \mathrm{~h}$ intervals and monitored with a differential scanning calorimeter. The test results indicated that MGDG and DGDG significantly retarded the polymorphic transformation from Form V to VI on cocoa butter compared with the control, with the addition of MGDG being the most effective. These results indicate that MGDG and DGDG extracted from various natural materials are effective in retarding the polymorphic transformation of cocoa butter.
\end{abstract}

Keywords: cocoa butter, glyceroglycolipid, polymorphic transformation, DSC

The formation of fat bloom on the surface of chocolate has been an industrial problem in chocolate manufacturing because it damages the product and significantly decreases the commercial value. Cocoa butter in chocolate is polymorphic, and has six forms (Form I, II, III, IV, V and VI), as identified by Wille and Lutton (1966). It is believed that fat bloom is formed due to the polymorphic transformation on cocoa butter. Thus, it is necessary to control the polymorphic transformation from Form V to VI to inhibit the fat bloom (Jana \& Thakar, 1993; Hartel, 1999). Control of this transformation has been proposed, for example, by adding a substitute fat highly compatible with cocoa butter (Lohman \& Hartel, 1994) or adding an emulsifier as a fat bloom inhibitor (Aronhime et al., 1988; Suwa \& Matsuda, 1993).

In our previous report (Nakae et al., 2000a, b), we described the retardation effect on the polymorphic transformation from Form V to VI of cocoa butter with the addition of monogalactosyldiacylglycerol (MGDG) extracted from pumpkin. We also showed that dark and milk chocolates to which the glycolipid fraction had been added containing MGDG inhibited fat bloom development in comparison with that of control (no addition) and lecithin.

The glyceroglycolipids are widely distributed in edible plants such as cereals, legumes, vegetables and fruits; their structures are shown in Fig. 1. Glyceroglycolipids consist of hydrophilic carbohydrate groups and hydrophobic fatty acid moieties which bind to a glycerol moiety, and are known to be some of the major components of biomembranes (Kates, 1990). They are also believed to play important roles such as enhancing membrane stability (Chapman et al., 1983). Some glyceroglycolipids have

E-mail: nakae-takashi@glico.co.jp also been reported to exhibit anti-tumor promoting activity (Murakami et al., 1995) or biological activities (Katsuoka et al., 1990; Sakata \& Ina, 1983).

In this report, we prepared MGDG, digalactosyldiacylglycerol (DGDG) and trigalactosyldiacylglycerol (TGDG) from various natural materials. Among the sources investigated were pumpkin purée, carrot purée, spinach purée, corn purée, wheat flour, soybean (lecithin), rice, barley, orange (flavedo), tea, cacao (raw seed), almond (raw seed) and Chlorella pyrenoidosa. We investigated the performance of their gained glyceroglycolipids as a dynamic controller of the polymorphic transformation of cocoa butter.

MGDG, DGDG and TGDG were prepared by the previously reported method of Nakae et al. (1998). They were confirmed by thin-layer chromatography (TLC) (Merck, art. 5626, Darmstadt, Germany) using chloroform-methanol- $\mathrm{H}_{2} \mathrm{O}(65: 25: 4, \mathrm{v} / \mathrm{v} / \mathrm{v})$, and were detected by spraying $50 \%(\mathrm{v} / \mathrm{v}) \mathrm{H}_{2} \mathrm{SO}_{4}$ followed by heating at $130^{\circ} \mathrm{C}$ for $5 \mathrm{~min}$. Each purity of extracted glyceroglycolipid was confirmed by high performance liquid chromatography (HPLC) using DIOL column $(4.6 \times 250 \mathrm{~mm}$, Merck) eluted with $n$-hexane/2-propanol/1-butanol/water $(60 / 30 / 7 / 3, \mathrm{v} / \mathrm{v} / \mathrm{v} / \mathrm{v})$ at a flow rate of $1.0 \mathrm{ml} / \mathrm{min}$ at $25^{\circ} \mathrm{C}$, with detection by an evaporative light scattering detector (ELSD; DDL-31, EUROSEP Instruments, Cergy, France). Detector temperature was at $60^{\circ} \mathrm{C}$ and the inlet nebulizer pressure was set to 1.5 bar.

Table 1 summarizes the composition of glyceroglycolipids as determined by the HPLC-ELSD method, which was significantly different among the sources examined. Corn, spinach, tea and pumpkin contain much MGDG and DGDG, while TGDG was present in pumpkin alone. Chlorella pyrenoidosa contains a great deal of MGDG. 


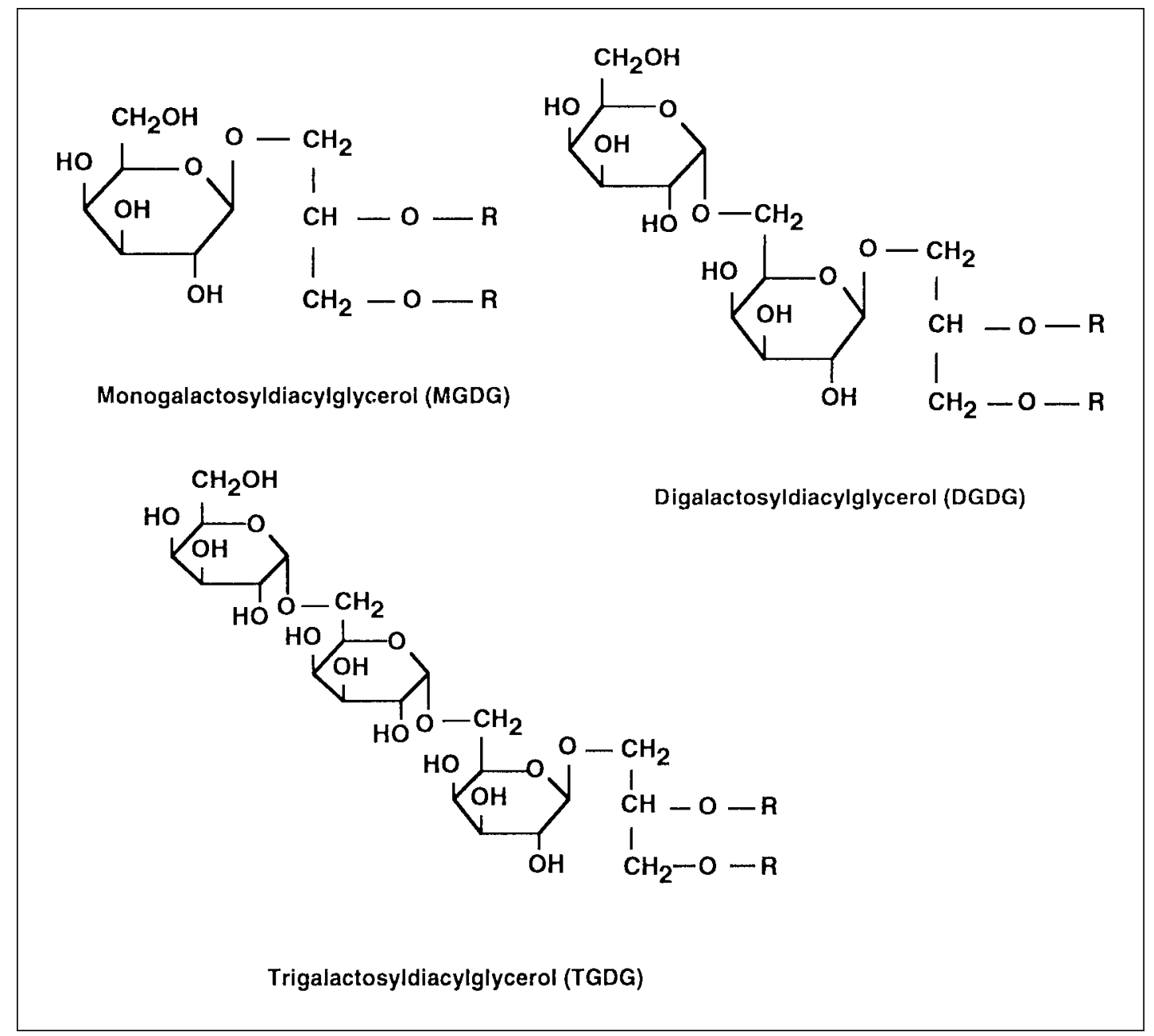

Fig. 1. Structures of MGDG, DGDG and TGDG. R represents acyl residue.

Table 1. Glyceroglycolipid compositions of various natural materials as determined by HPLC-ELSD.

\begin{tabular}{lccc}
\hline & MGDG & DGDG & TGDG \\
\cline { 2 - 4 } & Yield & Yield & Yield \\
& $(\mathrm{mg} / 100 \mathrm{~g}$ D.W. $)$ & $(\mathrm{mg} / 100 \mathrm{~g}$ D.W. $)$ & $(\mathrm{mg} / 100 \mathrm{~g}$ D.W. $)$ \\
\hline Barley & 14 & 60 & ND \\
Corn & 341 & 516 & ND \\
Rice (milled) & 0.2 & 0.7 & ND \\
Rice (bran) & 8.4 & 2.4 & ND \\
Wheat fiour & 102 & 225 & ND \\
Soybean (lecithin) & ND & 60 & ND \\
Cacao (raw) & ND & 58 & ND \\
Almond (raw) & ND & 1.6 & ND \\
Spinach & 840 & 878 & ND \\
Tea & 810 & 736 & ND \\
Carrot & 91 & 112 & ND \\
Pumpkin & 350 & 420 & 307 \\
Orange (flavedo) & 21 & 42 & ND \\
Chlorella pyrenoidosa & 3120 & 270 & ND \\
\hline ND: nt detected & & &
\end{tabular}

ND: not detected

Sugawara and Miyazawa (1999) have quantified glycolipids from edible plant sources by HPLC using an evaporative lightscattering detector. They reported that DGDG is rich in barley, corn and wheat flour, and that MGDG and DGDG are rich in leek, parsley, perilla, spinach and pumpkin. Our results are in agreement with their findings. However, since the concentration of glyceroglycolipids in natural materials can vary tremendously depending on the cultivar, growth condition, stage of development and processing method, the data given in Table 1 do not imply universal glyceroglycolipid contents.

Preparations of the methyl esters from each glyceroglycolipid were done by the previously reported method of Nakae et al. (1998). The fatty acid compositions of glyceroglycolipids in carrot, corn, wheat flour and Chlorella pyrenoidosa are shown in Table 2. In all glyceroglycolipids, linoleic acid, linolenic acid, palmitic acid, oleic acid and stearic acid were the major components; palmitoleic acid was present in Chlorella pyrenoidosa alone. Carrot was rich in palmitic acid and stearic acid, while corn and wheat flour were rich in linoleic acid.

We examined the polymorphism of cocoa butter through a thermo-cycle test by the following methods. Ten grams of cocoa butter (Daito Cacao Co., Ltd., Tokyo) at $60^{\circ} \mathrm{C}$ was cooled to $30^{\circ} \mathrm{C}$ within $10 \mathrm{~min}$. Each glyceroglycolipid sample was added at $5 \%$ when the temperature of cocoa butter reached $30^{\circ} \mathrm{C}$. Then, $0.25 \%$ seed crystal $\left[\beta_{1}\right.$ stable crystal of SOS (1, 3-distearoyl-2oleoyl-glycerol); Fuji Oil Co. Ltd., Osaka] was added to make an uniform Form V crystal type. After the seeding, cocoa butter was agitated for $5 \mathrm{~min}$ at $30^{\circ} \mathrm{C}$ to homogeneously disperse the glyceroglycolipid and seed crystal. Cocoa butter in a conical tube $(28 \times 100 \mathrm{~mm})$ was immediately cooled and solidified at $15^{\circ} \mathrm{C}$ for 
Table 2. Fatty acid compositions of MGDG and DGDG from various natural materials.

\begin{tabular}{|c|c|c|c|c|c|c|c|c|c|}
\hline \multirow{2}{*}{\multicolumn{2}{|c|}{ Fatty acid }} & \multicolumn{2}{|c|}{ Carrot } & \multicolumn{2}{|c|}{ Corn } & \multicolumn{2}{|c|}{ Wheat flour } & \multicolumn{2}{|c|}{ Chlorella } \\
\hline & & MGDG & DGDG & MGDG & DGDG & MGDG & DGDG & MGDG & DGDG \\
\hline Palmitic acid & $(\mathrm{C} 16: 0)$ & 24.0 & 39.5 & 4.7 & 15.3 & 11.5 & 15.4 & 2.0 & 14.3 \\
\hline Palmitoleic acid & (C16:1) & ND & ND & ND & ND & ND & ND & 10.6 & 7.1 \\
\hline Stearic acid & (C18:0) & 14.2 & 7.7 & 0.5 & 1.3 & 7.2 & 4.3 & 0.9 & 5.6 \\
\hline Oleic acid & (C18:1) & 8.2 & 4.3 & 28.8 & 18.7 & 8.2 & 6.6 & 37.6 & 30.3 \\
\hline Linoleic acid & (C18:2) & 35.4 & 39.3 & 55.8 & 54.2 & 69.1 & 68.1 & 13.5 & 18.4 \\
\hline Linolenic acid & (C18:3) & 18.2 & 9.3 & 10.2 & 10.5 & 4.0 & 5.6 & 35.5 & 24.2 \\
\hline
\end{tabular}

ND: not detected

$15 \mathrm{~min}$ in a cooling box. Thereafter, the glyceroglycolipid samples were stored at $20^{\circ} \mathrm{C}$ for one day (aging). The thermo-cycle test was then administered using a thermostat chamber (SANYO INCUBATOR): one cycle involves heating at $32^{\circ} \mathrm{C}$ for $12 \mathrm{~h}$ and cooling at $20^{\circ} \mathrm{C}$ for $12 \mathrm{~h}$. The polymorphic transformation on cocoa butter was determined with a differential scanning calorimeter (SSC220C type: Seiko Instrument Co., Ltd.) at a scanning rate of $5^{\circ} \mathrm{C} / \mathrm{min}$ after the test. The melting point was defined as peak temperature of the DSC melting peak.

Figure 2 shows DSC melting peaks of samples with the addition of 5\% MGDG and DGDG prepared from carrot through the
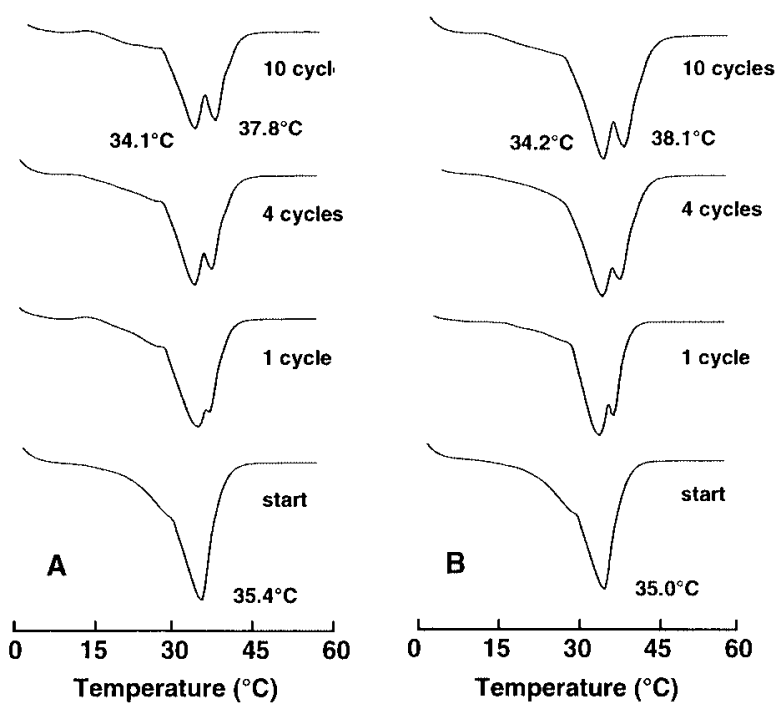

Fig. 2. DSC peaks of cocoa butter with the addition of $5 \%$ glyceroglycolipids of carrot through the $32 / 20^{\circ} \mathrm{C}$ thermo-cycle tests. A, MGDG; B, DGDG.
$32 / 20^{\circ} \mathrm{C}$ thermo-cycle tests. In the case of control (no addition of glyceroglycolipid), the solidified samples showed a large endothermic peak at $35.7^{\circ} \mathrm{C}$ which corresponded to the melting of Form V. In the cocoa butter including 5\% MGDG and DGDG prepared from carrot, corn, wheat flour and Chlorella pyrenoidosa, the solidified samples showed a large endothermic peak within 35.0 to $35.8^{\circ} \mathrm{C}$ which corresponded to the melting of Form V, respectively. From these results, the crystal lattices of cocoa butter were not affected by the addition of $5 \%$ glyceroglycolipids. After the 10 cycle tests of $32 / 20^{\circ} \mathrm{C}$, the control (no addition) sample showed a small melting peak of Form $\mathrm{V}$ at $33.8^{\circ} \mathrm{C}$ and a large melting peak of Form VI at $38.4^{\circ} \mathrm{C}$. Hence, the samples with the addition of 5\% MGDG and DGDG prepared from carrot have two melting peaks of Form V and Form VI which were almost equal. The samples with the addition of 5\% MGDG and DGDG prepared from wheat flour, corn and Chlorella pyrenoidosa also showed the same results. Table 3 shows the ratio between DSC melting peak area depending on Form V and Form VI of samples with the addition of 5\% MGDG and DGDG prepared from carrot, corn, wheat flour and Chlorella pyrenoidosa after 4 and 10 cycles of the $32 / 20^{\circ} \mathrm{C}$ thermo-cycle tests. After the 10 cycle tests of $32 / 20^{\circ} \mathrm{C}$, the control (no addition) sample showed $51.7 \%$ of Form V and $48.3 \%$ of Form VI. Hence, the sample with the addition of 5\% MGDG prepared from carrot showed $67.9 \%$ of Form V and $32.1 \%$ of Form VI, and that of DGDG prepared from carrot showed $66.9 \%$ of Form $\mathrm{V}$ and $33.1 \%$ of Form VI.

In our previous report (Nakae et al., 2000a), we described that the action of MGDG extracted from pumpkin for the retardation of the polymorphic transformation on cocoa butter was caused by the penetration of its molecules among triacylglycerols, and the incorporation of its molecules into created vacancies. We also described that the number of the acyl chain and galactose of

Table 3. Polymorphism of cocoa butter after thermo-cycle tests between 32 and $20^{\circ} \mathrm{C}$

\begin{tabular}{|c|c|c|c|c|c|c|}
\hline & \multicolumn{6}{|c|}{ Thermo-cycle condition $\left(32^{\circ} \mathrm{C} / 20^{\circ} \mathrm{C}\right)$} \\
\hline & \multicolumn{2}{|c|}{ Start } & \multicolumn{2}{|c|}{4 cycles } & \multicolumn{2}{|c|}{10 cycles } \\
\hline & Form V & Form VI & Form V 1 & Form $\mathrm{V}$ & Form V & Fonn VI \\
\hline Control (no addition) & 100 & 0 & 64.5 & 35.5 & 51.7 & 48.3 \\
\hline $5 \%$ MGDG (Carrot) & 100 & 0 & 73.3 & 26.7 & 67.9 & 32.1 \\
\hline 5\% DGDG (Carrot) & 100 & 0 & 72.8 & 27.2 & 66.9 & 33.1 \\
\hline $5 \%$ MGDG (Corn) & 100 & 0 & 69.3 & 30.7 & 61.8 & 38.2 \\
\hline $5 \%$ DGDG (Corn) & 100 & 0 & 65.7 & 34.3 & 59.7 & 40.3 \\
\hline $5 \%$ MGDG (Wheat flour) & 100 & 0 & 69.5 & 30.5 & 63.3 & 36.7 \\
\hline $5 \%$ DGDG (Wheat flour) & 100 & 0 & 66.1 & 33.9 & 54.9 & 45.1 \\
\hline $5 \%$ MGDG (Chlorella) & 100 & 0 & 69.3 & 30.7 & 62.7 & 37.3 \\
\hline 5\% DGDG (Chlorella) & 100 & 0 & 66.4 & 33.6 & 59.0 & 41.0 \\
\hline
\end{tabular}


glyceroglycolipid strongly influenced the polymorphic transformation on cocoa butter. In this report, MGDG and DGDG prepared from various natural materials significantly retarded the polymorphic transformation from Form V to VI on cocoa butter compared with the control (no addition). In particular, both MGDG and DGDG prepared from carrot had the greatest retardation effect on the polymorphic transformation of cocoa butter. The reason was postulated to be as follows. The fatty acid compositions of glyceroglycolipids in carrot were rich in palmitic acid and stearic acid. This means that the fatty acid compositions of glyceroglycolipids in carrot are similar to those of cocoa butter. Therefore, we suspect that glyceroglycolipids in carrot are structurally more compatible with cocoa butter than those of other materials.

On the other hand, MGDG tested in this study had a greater retardation effect on the polymorphic transformation of cocoa butter than DGDG. MGDG prepared from wheat flour, in particular had the effect, but DGDG had little. These results support our previous findings that the number of galactose of glyceroglycolipid influences the retardation effect on polymorphic transformation of cocoa butter because the fatty acid compositions of MGDG in wheat flour are similar to those of DGDG.

From these results, we concluded that MGDG and DGDG extracted from various natural materials were also applicable to retardation of the polymorphic transformation of cocoa butter.

\section{References}

Aronhime, J.S., Sarig, S. and Garti, N. (1988). Dynamic control of polymorphic transformation in triglycerides by surfactants: The button syndrome. J. Am. Oil Chem. Soc., 65, 1144-1150

Chapman, D.J., De-Felice, J. and Barber, J. (1983). Influence of winter and summer growth conditions on leaf membrane lipids of Pisum Sativum L. Planta, 157, 218-223.

Hartel, R.W. (1999). Chocolate: Fat bloom during storage. Manuf.
Confect., 79, 89-99.

Jana, A.H. and Thakar, P.N. (1993). Fat bloom in chocolates and confectionery coatings. Indian Food Ind., 12, 33-39.

Kates, M. (1990). In "Handbook of Lipid Research, vol.6," ed. by Plenum, New York, pp. 1-122, 235-320.

Katsuoka, M., Ogura, C., Etoh, H., Sakata, K. and Ina, K. (1990). Galactosyl- and sulfoquinovosyldiacyl glycerols isolated from the brown algae, Undaria pinnatifida and Costaria costata as repellents of the blue mussel, Mytilus edulis. Agric. Biol. Chem., 54, 30433044.

Murakami, A., Nakamura, Y., Koshimizu, K. and Ohigashi, H. (1995). Glyceroglycolipids from Citrus hystrix, a traditional herb in Thailand, potently inhibit the tumor-promoting activity of 12-O-tetradecanoylphorbol 13-acetate in mouse skin. J. Agric. Food Chem., 43, 2779-2783.

Nakae, T., Kometani, T., Nishimura, T., Takii, H. and Okada, S. (1998). Foaming power and emulsifying properties of the hydrolyzates by lipase from Rhizopus arrhizus on digalactosyldiacylglycerol and trigalactosyldiacylglycerol extracted from pumpkin. Food Sci. Technol. Int. Tokyo, 4, 235-240.

Nakae, T., Kometani, T., Nishimura, T., Takii, H. and Okada, S. (2000a). Preparation of glyceroglycolipids from pumpkin and their effects on polymorphic transformation of cocoa butter. Food Sci. Technol. Res., 6, 263-268.

Nakae, T., Kometani, T., Nishimura, T., Takii, H. and Okada, S. (2000b). Effect of glycolipid fraction on fat bloom in dark and milk chocolates. Food Sci. Technol. Res., 6, 269-274.

Sakata, K. and Ina, K. (1983). Digalactosyldiacylglycerols isolated from a brown alga as effective phagostimulants for a young abalone. Agric. Biol. Chem., 47, 2957-2960.

Sugawara, T. and Miyazawa, T. (1999). Separation and determination of glycolipids from edible plant sources by high-performance liquid chromatography and evaporative light-scattering detection. Lipids, 34, 1231-1237.

Suwa, N. and Matsuda, K. (1993). The anti-blooming effect of sucrose polyesters on chololates. Mitsubishi Kasei $R \&$ D Review, 7, 8-15 (in Japanese)

Wille, R.L. and Lutton, E.S. (1966). Polymorphism of cocoa butter. $J$. Am. Oil Chem. Soc., 43, 491-496. 